\title{
DiscoverFriends: Secure Social Network Communication in Mobile Ad Hoc Networks
}

\author{
Joshua Joy* \\ UCLA \\ Eric Chung* \\ UCLA \\ Zengwen Yuan
UCLA \\ Leqi Zou \\ UCLA \\ Jiayao Li
UCLA \\ Mario Gerla \\ UCLA \\ jjoy@cs.ucla.edu chung.yanhon@gmail.comzyuan@cs.ucla.edu zlqiszlq@cs.ucla.edu likayo@ucla.edu gerla@cs.ucla.edu
}

\begin{abstract}
Index Terms-Mobile communication, Ad hoc networks, Social computing, Security,

Abstract-This paper presents a secure communication application called DiscoverFriends. Its purpose is to securely communicate to a group of online friends while bypassing their respective social networking servers under a mobile ad hoc network environment. DiscoverFriends leverages Bloom filters and a hybrid encryption technique with a self-organized publickey management scheme to securely identify friends and provide authentication. Additionally, DiscoverFriends enables anonymous location check-ins by utilizing a new cryptographic primitive called Function Secret Sharing. Finally, to the best of our knowledge, DiscoverFriends implements and evaluates the first Android multi-hop WiFi direct protocol using IPv6.
\end{abstract}

\section{INTRODUCTION}

Mobile devices (e.g. smartphones and tablets) have become a rising platform for Online Social Networks (OSNs). Facebook, for example, reported over 1 billion mobile users [1] in the first quarter of 2014. Why are so many users moving to the mobile platform? One possible reason is that users can have instantaneous access to OSN with mobile devices at any time and place. But more importantly, users are attracted by the various interesting features that are only available on mobile platforms. Location-based services (LBS) [16] is one of the top features exploited by mobile users.

With GPS integrated into smartphones, mobile OSN users can easily localize themselves, which allows them to share their own location and related experiences with friends (e.g., the "Check-in" feature of the Facebook mobile app). Since it does so, users are more able to find nearby friends based on their locations. Therefore, the LBS functionality provides great convenience for users' social activities.

However, the application of LBS in OSNs also incurs some concerns surrounding the issue of privacy. When a user activates the LBS function in an OSN application, it exposes the user's location to the OSN service provider. In other words, Facebook can track the user's activity with this location information. One question is: Can the OSN users still utilize partial LBS without reporting their location to OSN providers. Typically, can we find nearby friends without requesting them from the Facebook servers. This paper provides a solution to this problem.

In this work, a Wi-Fi-based solution called DiscoverFriends helps an OSN user find nearby friends. The core idea is to leverage the local Wi-Fi communication to directly discover an OSN friend while bypassing OSN servers. The design faces two main challenges. The first problem is how to authenticate

*Eric and Joshua contributed equally to this work. an OSN user without going to the OSN server. Second, due to the broadcast nature of wireless communication, the messages can be overheard by other users. The potential eavesdropping can be a new threat to the privacy. To this end, DiscoverFriends addresses these issues using a Bloom filter-based approach with hybrid encryption to provide a higher level of security.

The solution provided by DiscoverFriends is not only limited to discovering nearby friends. It can further be employed to setup communication between friends in the same local Wi-Fi. In other words, OSN users can exchange multi-hop text messages and other data without going through the OSN server, which further helps to prevent users from being tracked by those OSN providers.

Finally, DiscoverFriends provides users a mechanism to anonymously "Check-in" their location on an OSN. To hide their identity, a user could "Check-in" and broadcast their location under a pseudoynm (shared previously with friends). However, the OSNs can easily examine the authenticated connection and infer the true identity. Discoverfriends utilizes a new cryptographic primitive called Function Secret Sharing (FSS) that is robust against traffic analysis attacks. As long as there are more than two users announcing their location, it is not possible to infer which user posted a particular "Check-in". FSS scales well to large anonymity sets as FSS is asynchronous and uses an efficient PRG.

This paper is organized as follows. Section 2 begins by elaborating on the application design followed by implementation details presented in Section 3. Section 4 describes the risk analysis under two threat models. Performance evaluation in Section 5 is done against alternative schemes described in the related works in Section 6. Lastly, Section 7 summarizes the features of the application and concludes the paper.

\section{DESIGN}

In DiscoverFriends, any OSN user can serve as an Initiator and leverage Wi-Fi broadcast to send request messages in order to find a specified friend, hereby referred to as Target. If the target receives the request, the target will then send back an acknowledgement to the initiator. The request message is constructed using Bloom filters and a certificate to achieve confidentiality and authentication, respectively.

The solution proposed is built based on an important assumption: each OSN user has a confidential ID, which is only accessible to the user's friends. This ID is not necessarily the string that OSNs use to identify each user, but it can be an extra confidential string. For instance, Facebook provides a user with a public username and private ID. This assumption is reasonable since many OSNs have access control mecha- 


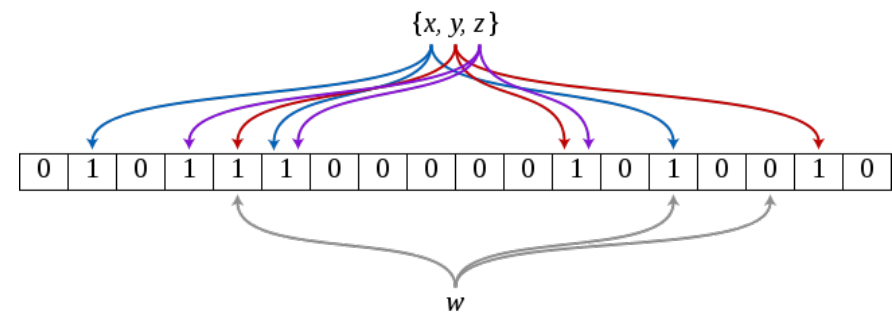

Fig. 1. Bloom filter representation where $x, y, z$ represent the elements in the set.

nisms, in which users can specify the accessibility of private information (e.g., whether it is public to all users or friends only).

Furthermore, DiscoverFriends is not limited to supporting only one OSN as it can utilize IDs from multiple OSNs to improve security. More specifically, users can XOR their IDs in Facebook and Google+ to generate a new ID. This new ID cannot be recognized by any single OSN provider even if the provider somehow captures the message. One drawback of this technique is that the intended friend has to be the initiator's friend on multiple OSNs, which may limit the usage of the application.

In this section, the application of Bloom filters is introduced and then explained how they are applied in DiscoverFriends. In addition to that, two encryption mechanisms are analyzed and a combined solution is selected to be used in the application. Moreover, an overview of key management techniques is provided, where one scheme is selected to be used in the application. Next, Function Secret Sharing (FSS) is introduced and explained how anonymous check-ins are achieved. Finally, the communication protocol is examined between the initiator and the target.

\section{A. Bloom Filter}

Bloom filter is a space-efficient probabilistic data structure, whose purpose is to test whether a specific element is in a given set or not. It may produce false positive results but will definitely not return false negative results. Bloom filter is widely used in caching mechanisms as well as security solutions.

Bloom filter is simply an $m$-bit array. When an element is added into a Bloom filter, $k$ hash function is applied on the element to get $k$ hash values in the range of $[0, m)$. Then, the corresponding bits in the array are set to 1 . When testing for an element, the same set of hash functions are applied before checking to see whether the corresponding bits in the Bloom filter are set to 1 . If all of them are 1 , then it means that the given element is included in the set; otherwise, it is excluded in the set. This explanation can be shown in Figure 1

When designing the Bloom filter, one focus is to optimize the number of hash functions $k$ and the length of the Bloom filter $m$, which can be calculated as a function of $n$, the number of inserted items, and $p$, the desired false positive probability as shown in Equation 1. In the case of DiscoverFriends, $n$ is the number of targeted friends the initiator wants to communicate to. Also, the choice of a small $p$ value is necessary as it corresponds to the probability that an attacker may be able to ascertain the hash of the initiator's ID. Subsequently, the appropriate $k$ value can be computed using $m$ and $n$ using Equation 2] [2].

$$
\begin{gathered}
m=-\frac{n \ln p}{(\ln 2)^{2}} \\
k=\frac{m}{n} \ln 2
\end{gathered}
$$

In DiscoverFriends, Bloom filters serve as the container of the IDs for friends. More specifically, when the initiator, who will be called Alice, sends the initial request, she adds the target's ID into a Bloom filter, which will be sent during the network initialization phase for friend discovery. Thus, when the target, say Bob, gets the request, he can determine whether or not the request is directed to him. In addition, Alice will also include her own ID in another Bloom filter carried in the request, so the target can also figure out who the initiator is. The network initialization phase does not need to be done over a secure channel as the Bloom filters provide the required level of security.

By using this technique, OSN users are not required to put their confidential ID into the broadcast message, which can be listened to by other people. Therefore, even if an adversary, Eve, eavesdrops the request message, it is extremely difficult for her to infer the initiator's ID if she is not the initiator's friend because the adversary only knows the hash values and cannot acquire the accurate corresponding ID with the hash values, let alone understand the user corresponding to that ID. However, it is important to note that although the use of Bloom filters can speed up initiator ID discovery by using fast hash algorithms to encrypt the confidential IDs and reduce latency by passing the lightweight Bloom filters over the network, it makes it easier for non-friends to discover the ID. Thus, security is traded off at the expense of lower discovery latency.

\section{B. Digital Signature and Key Management}

To ensure the security of the application, the idea was to use certificates for authentication. In order to generate a public key certificate, two schemes exist: public key infrastructure (PKI) and web of trust. In PKI systems, a certificate authority (CA) issues certificates that binds public keys to identities, and this information is kept in a central repository. On the other hand, a web of trust entails identity-based cryptography through self-signed certificates, where a key generation center (KGC) generates users' private keys. Evidently, this scheme is highly susceptible to man-in-the-middle attacks.

However, things are not that simple in an infrastructure-less model. Because of the ad hoc nature of DiscoverFriends, conventional public key infrastructure and web of trust schemes are less suitable for the application due to complicated certificate management [26]. Also, certificate authentication becomes impractical. The problem becomes clear when nodes cannot guarantee their connectivity and cannot rely on infrastructure to detect compromised mobile nodes [19]. In addition, certificate revocation in MANETs [25] becomes an issue because of the lack of centralized repositories and trusted authorities. 


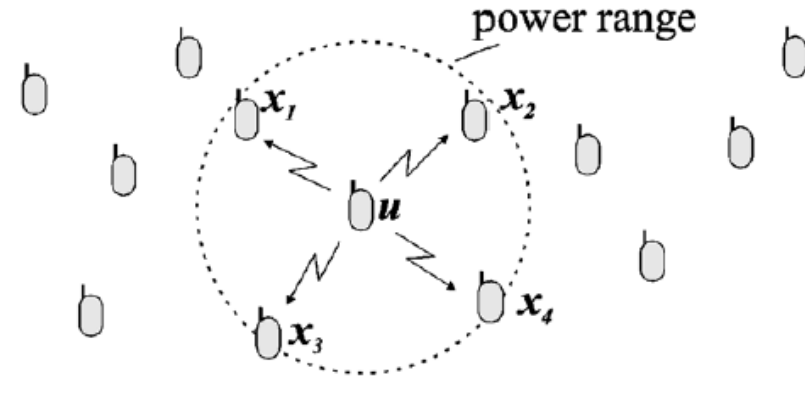

Fig. 2. Architecture

There are two main types of public-key cryptography: certificate-based cryptography (CBC) and ID-based cryptography (IBC). Previous works [33] have attempted to use CBC in MANETs. A naive approach for key distribution occurs in the network setup phase, where every node is preloaded with all other's public key certificates. However, as mentioned above, key management becomes an issue as key updates need to be handled in a cost-effective manner [14]. As a result, an improvement [6] using self-organized public-key management was built on the idea of a certificate chain. In a different angle, IBC eliminates the need for public-key distribution and certificates altogether by using a public key based off a public string identifying an individual. The underlying idea of IBC-based certificateless public-key management schemes is to have a set of network nodes share a master-key generated by threshold cryptography and collaboratively issue ID-based private keys. Built on top of the underlying concept, variations on the designing of certificateless public keys have come about in recent literature [35], [34], [36]. The downside of IBC is if the threshold number of nodes gets compromised, the network is breached.

DiscoverFriends uses a self-organized public-key management approach. Further details can be read up on in [27]. Every node in the network contains the following:

- Key repository (KR): Stores public keys sent by neighbor node.

- Shared key repository (SKR): Stores public keys of all nodes from the KR.

- Certificate repository (CR): Stores valid self-signed certificates.

In order to assess the security in the formation of trust, the network initialization phase plays an important role. The main objective of this phase is to distribute all the public keys to every node in the network. Instead of a certificate graph, a trust graph (TG) is generated by the shared key repository per node. At the end of the initialization phase, this trust graph is stored as a master graph (MG), which facilitates frequent public key updates.

In its reduced form, DiscoverFriends' model can be simplified as target nodes do not serve as intermediary nodes for other target nodes as shown in Figure 2. However, during prolonged application usage, it may be energy-efficient [5], [30] to pass group ownership to a target node (i.e. $x 1$ ) once the secure environment has been set up. This sets the need

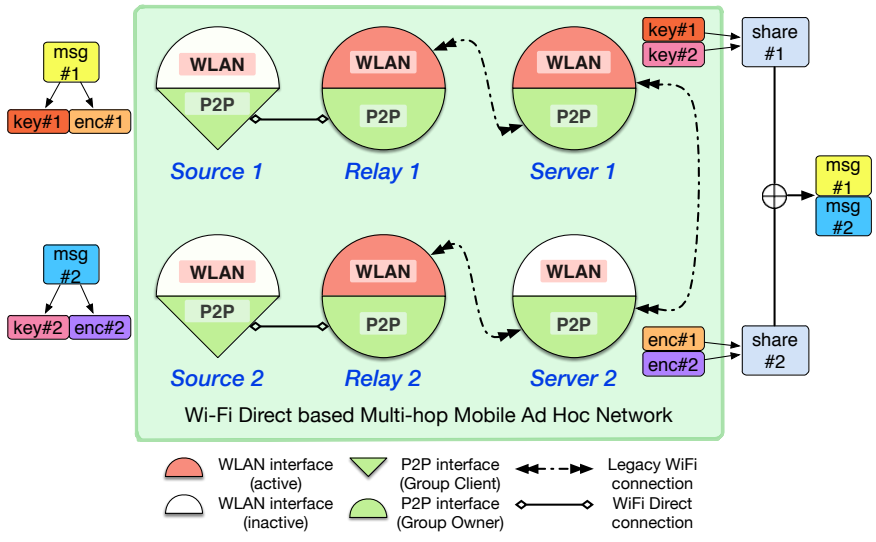

Fig. 3. Multi-hop Wi-Fi Direct routing

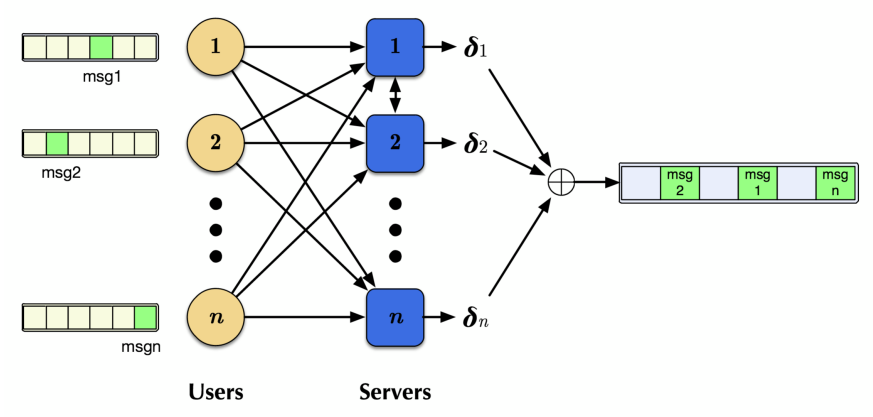

Fig. 4. Function Secret Sharing

for an efficient key management scheme specifically designed for MANETs. In this case, the target node must know the certificate and public key associated with all nodes within its group. Therefore, the TG provides a path to the end node, which has the corresponding private key to decrypt the sender's message. Within the data structures of each node, valid certificates and public keys of all other nodes in the group are contained. The transmission of the information is done during the network initialization phase, which in the case of DiscoverFriends, happens between friend discovery and communicating to the group of nodes.

\section{Anonymous Check-in}

Our goal for anonymity is that a user is able to transmit a message such that the message is unable to be linked back to the user. We assume that each server is operated by a separate OSN and there is at least one honest OSN.

We utilize Function Secret Sharing (FSS) [3] to anonymously transmit a message that is protected against traffic analysis as long as there is at least one honest server. The intuition behind FSS is as follows. Suppose there is a database with $2^{n}$ indexes. Each user is able to write their respective message to a randomly selected database index. If each user randomly selects an index and writes to the randomly chosen index, an adversary will not be able to infer which database index a particular user has written to. Thus, FSS defends against traffic analysis. As long as there is one honest server, the remaining servers will not be able to combine their shares 
to reconstruct the original message. Each user XORs their message to generate the shares such that the XOR sufficiently randomizes the bits.

We now explain FSS in further detail. Suppose we wish to secretly share a function with $p$ parties where at least one party is honest. Suppose there is an input $x$ which is $n$ bits and the output $y$ which is $m$ bits. Given $p$ keys such that the strings are randomly sampled from the space of $\{0,1\}^{2^{n} * m}$ (total number of inputs multiplied by the size of the message), these strings should evaluate to the message $m$ whereby $f(x)=y$ such that $\bigoplus_{i=j}^{p} k_{i}[x]=y$. Thus, in this case $p-1$ parties are unable to XOR their keys to discover $f(x)$.

As long as two or more users do not choose the same input $\mathrm{x}$ (we assume no collisions), each user is able to write their respective message $\mathrm{y}$ to input $\mathrm{x}$. Each user proceeds by sending their keys to each respective server. Each server then performs a bitwise XOR of the evaluation of every $f\left(x_{k}\right)$ over the received key $k$ such that $\bigoplus_{x=0}^{2^{n}-1} f\left(x_{k}\right)$. That is there is a total of $2^{n}$ evaluations at each server for each key. Each result of $f\left(x_{k}\right)$ is XORed locally at each server resulting in an intermediate computation. This results in an intermediate computation which at the end of the agreed epoch, each server then shares with each other.

Intermediate Results:

$$
\begin{aligned}
& \delta \text { shares server } 1=\bigoplus_{x=0}^{2^{n}-1} f\left(x_{k_{1}}^{a}\right) \oplus f\left(x_{k_{1}}^{b}\right) \ldots \oplus f\left(x_{k_{1}}^{p}\right) \\
& \delta \text { shares server } 2=\bigoplus_{x=0}^{2^{n}-1} f\left(x_{k_{2}}^{a}\right) \oplus f\left(x_{k_{2}}^{b}\right) \ldots \oplus f\left(x_{k_{2}}^{p}\right)
\end{aligned}
$$

Final Output:

$$
\begin{aligned}
& \text { server }_{1}=\delta \text { server }_{1} \oplus \delta \text { server }_{2} \\
& \text { server }_{2}=\delta \text { server }_{2} \oplus \delta \text { server }_{1}
\end{aligned}
$$

\section{Servers' Output Should Match}

$$
\text { server }_{1}=\text { server }_{2}=\left\{y_{0}, y_{1}, \ldots y_{2^{n}-1}\right\}
$$

For our purposes, the particular function we are interested in is a distributed point function. A distributed point function (DPF) maps an input $x$ to output $y$ such that $\mathrm{f}(\mathrm{x})=\mathrm{y}$ and $\mathrm{f}\left(\mathrm{x}^{\prime}\right)=0$ for all $\mathrm{x}^{\prime} !=\mathrm{x}$. Function secret sharing for $p$ parties allows to secretly share a DPF amount $p$ parties such that the function is unable to be reconstructed with p-1 keys and is thus collusion resistant as long as there is one honest party.

The FSS algorithm works as follows. We consider the special input $x$ as a grid where the rows are indexed by the first bits and the columns are indexed by the second bits. The two dimensional grid allows a reduction in the key length to achieve $O\left(2^{n / 2} \cdot 2^{n / 2} \cdot m\right)$.

Next, the function must be cryptographically hidden. To achieve this, $2^{p-1}$ random seeds are chosen for each row. Additionally, $2^{p-1}$ correction words are chosen such that for the special row (the first bits of the input $x$ ) XOR together equal the message as the specific point and zeros elsewhere.

The end result should be that the seeds for each row will cancel each other when XORed together, leaving only the special row. To achieve this, special binary arrays are formulated to be multiplied by the seeds to ensure that an even number of the seeds appears in order to cancel each other out except for the special row. To construct the binary arrays, for all rows (except the special row) an even bit array is formulated such that there are $p$-bit columns such that the number of 1 bits is even. For the special row an odd bit array is formulated such that there are $p$-bit columns such that the number of 1 bits is odd.

\section{Communication Protocol}

The communication protocol consists of two parties: Initiator and Target. The first two stages represent the network initialization phase. In the first stage, the initiator, who wants to discover a certain friend (target), sends a Wi-Fi broadcast request consisting of three parts:

- $B F_{c}$ : The Bloom filter containing the Target's ID.

- $B F_{c}+: B F_{c} \oplus$ hash of Initiator's ID.

- $C F$ : Initiator's certificate encrypted with AES; the encryption key is the hash of the Initiator's ID.

Here, $B F_{c}$ is used to identify whether the target is a person of interest in the invitation. $B F_{c}+$ builds on top of this knowledge and helps the target identify the initiator. Lastly, $C F$ is used for authentication without going through the OSN server. In detail, the certificate contained inside is self-signed by the initiator.

In the second stage of the protocol, the target receives the request message and proceeds in the following steps:

1) Test whether the Target's own ID is in $B F_{c}$. If not, terminate the procedure. Otherwise, proceed to next step.

2) $B F_{c} \oplus B F_{c}+\rightarrow$ hash of Initiator's ID.

3) Traverse the Target's own friend list and apply hash functions to each friend's ID to determine if a match with the Initiator's ID exists.

4) Once a match has been found, take the hash of Initiator's ID to decrypt $C F$.

5) Check the validity of the certificate in $C F$ and finish authentication.

6) If the Target accepts the invitation from the Initiator, the Target replies with own certificate encrypted with AES; the encryption key is the hash of the Initiator 's ID.

In stage three, once all peers are connected, the Initiator sends the set of certificates to the connected peers. Step 6 and stage three are necessary for the Initiator to update the SKR and CR of other targets that are connected to the Initiator such that after the network initialization phase, proper key management is ensured and eavesdropping attempts are averted. Assuming all targets who want to connect to the Initiator are connected, the network initialization phase ends. This leads into stage four and stage five, which represent subsequent communication. Optionally, step 6 is repeated as many times as necessary during network connection in order to push certificate updates to the group as certificates may expire during the communication session. These two stages may be 
repeated multiple times until the connection is broken. In stage four, the Initiator communicates to the Target as follows:

1) Generates a random symmetric encryption key.

2) Uses symmetric key to encrypt a message.

3) Encrypts symmetric key using target's public key.

4) Broadcasts the encrypted message and encrypted key.

In response, the Target replies in the following manner:

1) Uses own private key to decrypt the symmetric key.

2) Uses symmetric key to decrypt the message.

3) Sends back an AES-encrypted message to Initiator.

Now, the initiator and target are able to communicate directly, effectively bypassing OSN servers.

\section{IMPLEMENTATION}

In this section, details of the implementation are provided, including the Bloom filter, Wi-Fi communication, and OSN adaptors.

Bloom Filter One important issue of implementing Bloom filters is the usage of the hash function. There are several candidates, including murmur, fvn series of hashes, Jenkins Hashes, etc. Not only should the ideal hash function provide independent and uniformly distributed results, it should also be as fast as possible to account for the mobile platforms' limited computation capabilities. Therefore, the final choice should preferably avoid widely used hashing algorithms, which generally run slow. After considering different factors, murmur32 was selected as the prime hash function for DiscoverFriends and the parameter to reduce the false positive probability was tuned to $2 \%$.

Encryption For symmetric key encryption, DiscoverFriends uses AES encryption. In the network initialization phase, the secret key is the SHA-1 hash of the initiator's ID, trimmed to use only the first 128 bits. During subsequent message exchanges, the secret key is randomly generated using SHA1PRNG. Also, for this scheme, the cipher uses a PKCS5Padding transformation, which supports AES 128-bit keys.

As for asymmetric cryptography, a public/private key pair is generated using a RSA algorithm, in which key sizes are 1024 bits. With this key pair, DiscoverFriends generates self-signed X.509 certificates using the sun.security.x509 package.

Wi-Fi Broadcast To implement Wi-Fi broadcast on Android, the IP address of the Wi-Fi interface is firstly retrieved. Then, the corresponding Wi-Fi broadcast address is derived. Afterwards, a UDP socket is established to send broadcast messages.

\section{A. Wi-Fi Direct}

First, a brief introduction of Wi-Fi Direct is necessary, followed by a discussion on the choice and effectiveness of this technology. The main purpose of Wi-Fi Direct is to enable peer-to-peer connections without requiring a wireless access point (AP), allowing both one-to-one and one-to-many connections among different devices through Wi-Fi speeds. To enable compatibility for older devices using this newer technology, only one device needs to be certified to the protocol to allow for communication. In addition, this technology enables connection establishment with any device discovered within a 200 meter radius, thereby leveraging the concept of locality.
To introduce the reasoning behind choosing to utilize Wi-Fi Direct technology over other methods, two general scenarios where DiscoverFriends may be used are examined: a user is connected to a local area network and a user is not connected to one. Lastly, this section ends with an analysis of the applicability of the technology.

1) Connected to $L A N$ : For the first scenario, the user is connected to a LAN, so we utilize Android's Network Service Discovery to detect other devices that can service DiscoverFriends. After detecting which devices are currently running the application, a UDP broadcast is sent to these devices. However, a few devices such as some HTC devices block these broadcasts, so a workaround for these exception cases is needed. Here, Wi-Fi Direct is not necessary because of the presence of a wireless network backbone. Additionally, helper nodes can be utilized to help to overcome the single hop limitation. The helper nodes would need to pass on the initiator's Bloom filter for initial validation as well as any subsequent messages.

2) Not Connected to LAN: In a latter more probable scenario, the user enters a location where there is no network infrastructure available. For example, the user, Alice, goes to a park and wants to be able to communicate to all her friends there. Here, she can use the Wi-Fi Direct technology in DiscoverFriends to accomplish this goal through a two-step process.

1) The initiator discovers nearby devices and sets up the device, making the user the group owner.

2) Attempts to establish a one-to-many connection with all the discovered devices.

For the purpose of this application, the group owner is set to be the server and the connected devices to be the clients. Once connected, the initiator is able to communicate to all its connected clients using the channel managed by the WifiP2pManager. Wi-Fi Direct is suitable for DiscoverFriends' assumptions because it leverages the locality of this technology. However, all this assumes that the initiator, as well as the friends, have the Wi-Fi Direct functionality. If the friends do not support Wi-Fi Direct, then this technology is not suitable for the DiscoverFriends environment. Although the initiator can set up his phone as a wireless access point, the network name and passphrase to connect to the device, which is generated by Android's WifiP2pManager, are randomly generated. Also, note that how DiscoverFriends is designed, discovery of new friends is not permitted unless the network is reinitialized. This is not because of Wi-Fi Direct limitations but because of preventing replay attacks. Therefore, it is not possible for the initiator to provide his friends with the access information beforehand, thwarting Wi-Fi Direct's usefulness in the application.

3) Multi-Hop Capability: Wi-Fi Direct is the best option under the mobile ad hoc scenario, because of it makes multihop connection possible, which is another important merit and greatly extends the connectivity. Android is well known for not supporting the Wi-Fi Ad-Hoc (IBSS) mode (the Issue 82 on the Android Open Source Project has been unsolved since 2008 [13]), so users cannot form ad hoc connection using the Wi-Fi without rooting their phones.

Using Wi-Fi Direct to form multi-hop connection is concep- 

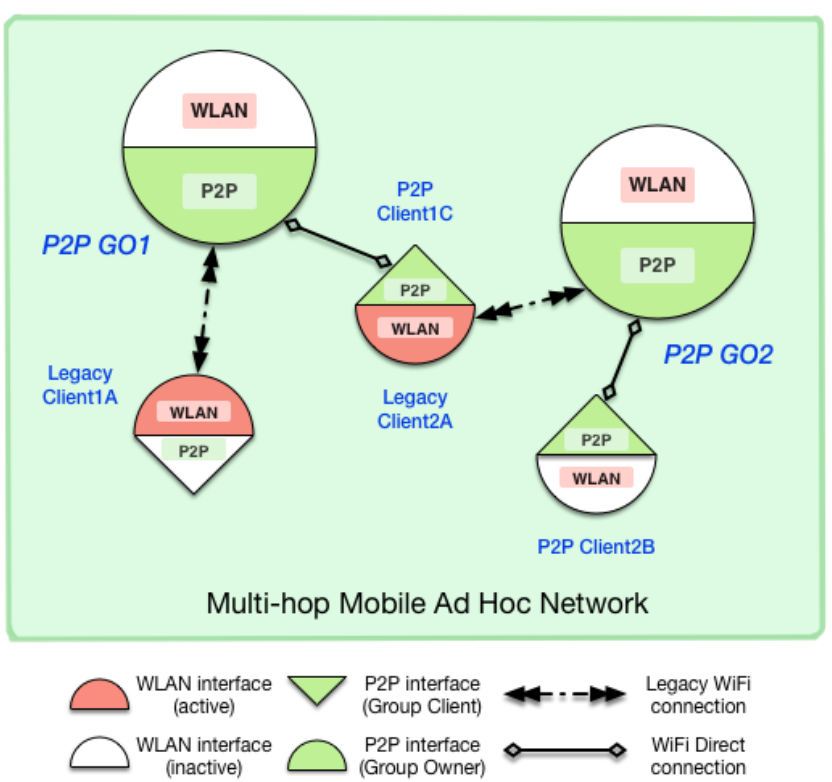

Fig. 5. Wi-Fi Direct multi-hop connection topology

tually straightforward. The device which support Wi-Fi Direct comes with two Wi-Fi interfaces: one is the traditional WiFi interface (legacy wlan interface) which is mainly used by regular WLAN connection, and the other is the Wi-Fi P2P interface (p2p-p2p or p2p-wlan interface) which is created for Wi-Fi Direct. Thus, with both the legacy wlan interface and Wi-Fi P2P interface available, we can configure the intermediate nodes to be relay nodes by receiving traffic on one interface and sending it out on another. The idea of multihop connection topology is shown in Figure 5. The node in the middle connects to Wi-Fi P2P Group Owner 1 (GO1) via its Wi-Fi P2P interface, and simultaneously connects to Wi-Fi P2P Group Owner 2 (GO2) via its traditional Wi-Fi interface. Thus, message can be transmitted from $\mathrm{GO} 1$ to $\mathrm{GO} 2$ via the relay node, which forms a 2-hops connection. Theoretically, the multi-hop connectivity is not limited, and can be extended to arbitrary number of nodes.

The logical equivalent of relaying the traffic on different interfaces is being able to configure routing table (or iptables, to be more accurate) in the runtime, which is easy enough for rooted devices. The real challenge here is to implement the relay topology without root privilege, which is crucial for our application to be useful for the majority of the consumers without breaking Androids security model. Though it seems impossible for commodity-off-the-shelf unrooted Android phones, we can use Android's public API to make such topology work. The key is Android's IPv6 support, which allow us to specify the network interface when sending out the packets, which perfectly solves connectivity issue.

Admittedly, there exists other workaround solutions to support multi-hop connection, for example, using the Bluetooth and Wi-Fi together [31]. However, the Bluetooth is not even a competitive alternative for Wi-Fi Direct, in terms of data rate, transmission range and security.
4) Applicability: Finally, the applicability and general drawbacks of Wi-Fi Direct is examined. Aside from compatibility with legacy devices, Wi-Fi Direct draws significant power from the Android device, so it is not feasible to keep Wi-Fi Direct on for an extended time [18], which even the system warns the user when tries to enable the feature. However, it is usable for DiscoverFriends because this application only runs for a short period of time to exchange short messages. In addition, the connection range is limited to 200 meters, but this is a suitable distance for the application.

\section{B. OSN Adaptors}

There were many OSN adaptors to choose from, where the main ones were Facebook, Google+, and Twitter. Here, DiscoverFriends implements the first two. To support dynamic changes in friendship, DiscoverFriends needs only to add a new entry to the Bloom filter. The updated Bloom filter would then be sent to all peers.

Facebook Facebook was integrated in DiscoverFriends in order to obtain a user's Facebook friends using Graph API v2.0. The Facebook IDs will then be placed into the initiator's request Bloom filter. Another Bloom filter is constructed by copying the previously constructed Bloom filter, which contains the hashed friends' IDs, and appending onto it, the user's own ID. Note the length of the friend list in the worst case for the expected insertions with a $2 \%$ false positive probability, the optimal size of the Bloom filter is decided using Equation 2

Google+ The solution is extended to support Google+. When using both OSNs, the two IDs representing a user is XORed to generate a new ID. This new ID can only be identified by the initiator and the target who is the initiator's friend on both OSNs. One issue is how to determine which friends meet this requirement. Currently, the solution is to hard code some common friends in the system. Later, it can be replaced by a smarter mechanism such as comparing the name and email to infer the potential common friend.

\section{Risk ANALYSIS}

Risk analysis is done on three potential forms of network attack: replay attack, eavesdropping by a common friend, and traffic analysis of "Check-ins". For the replay and eavesdropping attacks, let us consider the simplified scenario where Alice is the initiator who connects to Bob. For the traffic analysis attack we consider two or more users sharing their "Check-ins" with multiple OSNs.

\section{A. Replay Attack}

In the case of a replay attack, we assume an adversary is able to intercept and retransmit our application data. One purpose of this attack may be to fraudulently establish a subsequent connection between the adversary and the initiator's targeted group of friends for a malicious rendezvous. Consider now, an adversary, Eve, that is eavesdropping and wants to use that knowledge of the connection to communicate to Bob by posing as Alice. In order for Eve to execute a successful replay attack, she must firstly be authenticated by Bob.

For authentication, DiscoverFriends uses certificates which have a limited validity period. Limited validity period refers to 
a successful instance of meeting up. Therefore, a new instance will result in requiring a new certificate in that respect such that the old certificate will be deem void, stripping away the possibility of a replay attack. So if Eve tries to replay data that contains an expired certificate, Bob will know. Procedure for certificate revocation for the self-organized public-key management system is described in [27]. As a result, any attempts at replay attacks are thwarted.

Although man-in-the-middle attacks are prominent in selfsigned certificates as a user does not have the certificate in advance for validation, these malicious attempts are handled by proper detection of a compromised node using the trust graph in the chosen key management scheme. In other words, building a trust graph during the initial insecure network initialization phase helps enable users to determine valid certificates provided by the nodes contributing to the master graph. DiscoverFriends assumes that the social media site, initiator, and target are not compromised. This scheme's strong chain of trust also protects against Sybil attacks, where an attacker can take on multiple identities in attempt to subvert the reputation system of a peer-to-peer network. Therefore, selfsigned certificates by Eve will not be present in each node's $\mathrm{CR}$, successfully preventing man-in-the-middle attacks.

\section{B. Eavesdropping by a Common Friend}

When a common friend, Eve, eavesdrops, she is able to identify the initiator because the hashed ID of one of his friends will come out as a positive match. Without any additional security measurements, Eve is able to decrypt subsequent messages sent from Alice using AES with the matching ID from her friend list as the key. One such approach is that instead of using the ID of the initiator as the key for AES, the application uses the public key obtained from the certificate. In the case of DiscoverFriends, an additional security measurement is present as the AES key is encrypted using public-key cryptography. As a result, the communication between initiator and target will be private, preventing future eavesdropping.

It is also important to note that aside from common friends, bystanders may be able to guess who the initiator is. This leads to guessing the respective ID as it may be mnemonic such as a variation of the initiator's name. Similar to the case of the common friend, the extra security measurement of using the public key is necessary to hide the conversation.

\section{OSN De-Anonymizaton Attack}

Suppose an OSN which operates a server wishes to deanonymize a user and discover who posted a particular "Check-in" message. The user transmits a share to each OSN. As the user establishes an authenticated connection, each OSN knows which shares a user trasnmits. However, in order for a single OSN to reconstruct the message, all shares are required. A single OSN will not be able to reconstruct the original message as long as there is one honest OSN that does not collude.

When two or more users post their "Check-in", the shares will be XORed at each OSN. The XOR sufficiently randomizes the bits such that when two or more users participate, it is not possible to determine which user chose a particular database index. Thus, DiscoverFriends ensures users are able to anonymously post their "Check-in" as long as there are two or more users participating and there is at least one honest server that does not collude.

\section{Performance Evaluation}

\section{A. Experiment Setup}

To evaluate the performance of DiscoverFriends, we utilize Android 4.0+ devices. As mentioned earlier, although the application supports legacy devices, using any versions older than 4.0 will undermine the application's purpose as an Android randomly generated passphrase is needed to be known to Wi-Fi Direct unsupported phones.

Here, the network, computational, and storage costs are compared for three systems: DiscoverFriends' using hybrid encryption, DiscoverFriends using only AES with Bloom filters, and a system using prearranged ABE policy trees as the initial baseline. In the following two sections, it is shown that using Bloom filters and a hybrid encryption technique performs the best over the latter two systems.

\section{B. Multi-hop Network Cost}

We use four Android Nexus 7 tablets to evaluate the performance of multi-hop Wi-Fi Direct network. We connect tablets through the traditional Wi-Fi interface and the Wi-Fi P2P interface in a linear chain, as illustrated in Section III-A3 In general, a $n$-hops scenario consists of $n+1$ phones chained via $n$ Wi-Fi P2P groups. We start from the 1-hop scenario, where two tablets form a group. Both tablets are Wi-Fi P2P Group Owners and they connect via the traditional Wi-Fi interface. We use iPerf to test the performance of the multihop network. For the iPerf client, we direct the test traffic to a socket on localhost to our application. Then application on the source node tablet will read the data sent by iPerf and relay it to the destination node via the multi-hop network. When the destination node receives the data, it will redirect it to the iPerf server running on its localhost. For simplicity, we utilize UDP protocol to send the traffic. We measure the network throughput and packet loss by varying offered load. Together with throughput, packet loss can be utilized to estimate the maximum bandwidth. To evaluate a $(n+1)$-hops scenario, we add a tablet to the beginning of the $n$-hops chain, and connect it to the first tablet in the $n$-hops chain using the same connection topology.

Figure 6 shows that as expected the throughput decreases with the number of hops. This degradation is due to the wireless interference between each hop as the chain utilizes the same channel in order to communicate per the Wi-Fi Direct standard. The maximum observed throughput is about $19 \mathrm{Mbps}$ for the 1-hop scenario, about $18 \mathrm{Mbps}$ for the 2hops scenario, and about $10 \mathrm{Mbps}$ for the 3-hops scenario. The severe degradation between the 2-hops and 3-hops is due to the spatial diversity and proximity placement of the tablets.

Figure 7 depicts the packet loss as the number of hops are varied. As expected, the packet loss rapidly increases as we add load on the multi-hop network and first occurs at about 8 Mbps. Both the 1-hop and 2-hops incur 10\% packet loss at around $22 \mathrm{Mbps}$ and $20 \mathrm{Mbps}$ respectively. The packet loss for 
TABLE I

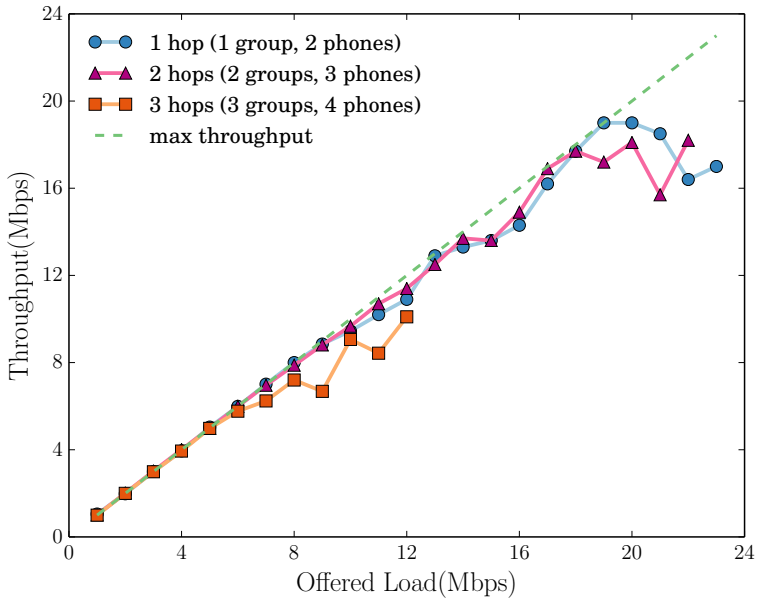

Fig. 6. Wi-Fi Direct multi-hop throughput.

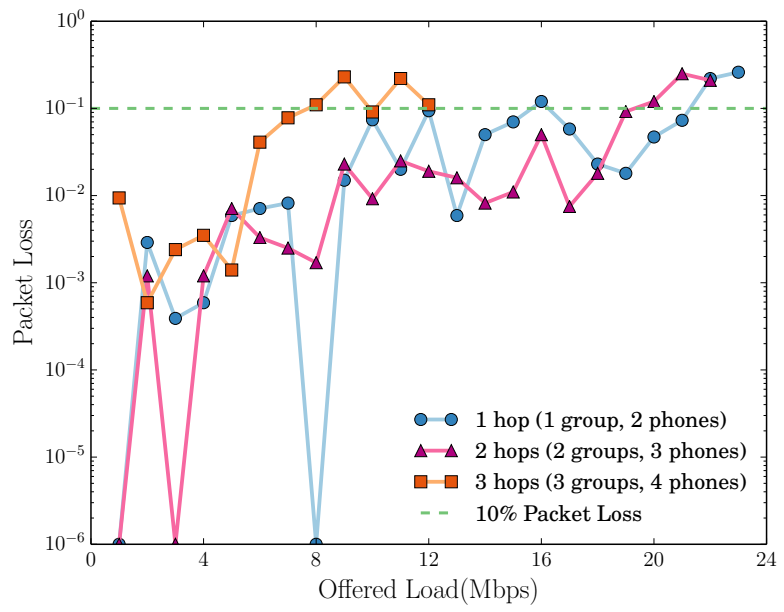

Fig. 7. Wi-Fi Direct multi-hop packet loss.

the 3-hops scenario is attributed to the wireless interference and tablet placement is with the throughput.

\section{Computational Cost}

Because encryption and decryption are computationally costly especially in a MANET environment, energy-efficient approaches in applications are necessary. The system using ABE keys has high computational cost due to its large keys versus DiscoverFriends using only AES with Bloom filters approach, which cuts down the cost to a fraction. However, this approach trades off security for efficiency. Here, security suffers due to by the small chance of false positives using Bloom filters, whereas the advantage of using $\mathrm{ABE}$ is its stronger protection scheme. The cost of encryption and key generation for $\mathrm{ABE}$ scales with the number of attributes and the complexity of the access policy. Note that performing offline key generation can speed up initial computational cost while encryption must be done online. Therefore, a common approach of using hybrid cryptosystems such as that in the final form of DiscoverFriends lowers computational cost by
Function Secret Sharing Server Processing Time

\begin{tabular}{|c|c|}
\hline Message Size & Server Processing Time \\
\hline 62 bytes & 8 seconds \\
\hline 125 bytes & 14 seconds \\
\hline 187 bytes & 20 seconds \\
\hline
\end{tabular}

TABLE II

KEYSTORE SIZE COMPARISON IN KB

\begin{tabular}{|c|c|c|}
\hline & 100 friends & 1000 friends \\
\hline Use of Bloom Filters & $4.52 \mathrm{~KB}$ & $4.52 \mathrm{~KB}$ \\
\hline No Use of Bloom Filters (e.g. ABE) & $44.9 \mathrm{~KB}$ & $449 \mathrm{~KB}$ \\
\hline
\end{tabular}

only using the slow asymmetric algorithm on keys rather than on messages while maintaining the necessary security features.

As Function Secret Sharing (FSS) relies on the XOR rather than any expensive pairing operations, FSS is computationally efficient. Table [I evaluates the computational overhead for an anonymity set size of 2048 (we expect that users rarely exceed having 1000 friends). We evaluate messages up to 187 bytes, which supports our message size of 160 bytes. We have implemented FSS using Go. The server (acting as an OSN) is a Linux server running Linux 3.11 .0 with a $2.2 \mathrm{GHz} \mathrm{CPU}$ with 24 cores and 32GB ram.

\section{Storage Cost}

The amount of storage used in DiscoverFriends is proportional to the subset of successfully connected friends. It is shown that DiscoverFriends' storage cost is significantly less than the other. In an experimentation, two initiators who have 100 and 1,000 friends, respectively only want to communicate to a subset of 10 friends. Table $\mathrm{II}$ depicts this experiment, and it is shown that DiscoverFriends' approach requires less storage space compared to the other approach. The reasoning behind this is because the Bloom filter prunes the initiator's full friend list to a targeted group of friends. However, not everyone in the list may be present or choose to respond to the initiator. As a result, only the keys corresponding to the connected friends are stored versus the entire set of $\mathrm{ABE}$ keys the other model has to store. Because ABE generates an ASK for each user, the number of keys corresponding to each user in an OSN may amount in the thousands, thus it is not practical. Furthermore, under this approach, the social network servers would know the keys and can defy attempts of covered rendezvous. From this, the effectiveness of using Bloom filters come to light as they only manage which users can decrypt the message using a bit array structure while cutting OSN servers out of the picture.

While FSS is computationally efficient, this computational efficiency has a trade-off of high memory consumption. The space overhead is proportional to the anonymity set size desired in order to sufficiently anonymize and obfuscate the users' responses. An anonymity set size of 2048 requires memory consumption of about $32 \mathrm{~GB}$ ram.

Another measurement was done on the sizes of DiscoverFriends' different types of network packets: setup, certificate update, and normal. In detail, the setup packet consists of the user's certificate and two Bloom filters, where the parameters for the number of expected insertions $n$ is 1000 and the false positive probability $p$ is 0.02 . The normal message has a 
TABLE III

TOTAL PACKET SIZES IN BYTES

\begin{tabular}{|c|c|}
\hline & Total packet size \\
\hline Setup & $2,516.59$ bytes \\
\hline FSS Share & $1,468,006$ bytes \\
\hline Certificate Update & 481 bytes \\
\hline Normal & 176 bytes \\
\hline
\end{tabular}

maximum limit of 160 characters, which gets encrypted using AES-128. These measurements are shown in Table [II

\section{RELATED WORK}

\section{A. Encryption Mechanisms}

Attribute-based encryption (ABE) is an alternative method of discovering friends in place of Bloom filters. Users of $\mathrm{ABE}$ create three different types of keys: ABE public key (APK), $\mathrm{ABE}$ master secret key (AMSK), and ABE secret key (ASK). The first two are generated on a user bases while the third is created per friend. The uses of these keys are summarized as follows: APK is for encryption, AMSK is for secret keys generation, ASK is for associating the users set of attributes. There are two types of ABE schemes: Ciphertext-Policy and Key-Policy. The Ciphertext Policy Attribute-Based Encryption (CP-ABE) consists of four steps: Setup, Encrypt, KeyGen, and Decrypt. Similar to Bloom filters, ABE targets a specific group that can decode the encrypted message. Through this scheme, only a subset of all users with attributes that match the access policy can decrypt the messages. Synchronization is enabled using a key chain mechanism. The second scheme, Key-Policy Attribute-Based Encryption (KP-ABE) is similar to the first scheme, consisting of the same four algorithms but differs on attribute association. Rather than associating the attributes with the user, KP-ABE instead associates with the plaintext message. In other words, the decryption policy enables only those users who match the ciphertext attributes, which are associated with a plaintext message. In addition, there are different flavors of ABE systems such as token-based $\mathrm{ABE}$ (tk-ABE) [15], which protects against key cloning.

\section{B. Multi-Hop Connectivity}

Multi-hop connectivity is well studied in the literatures [17], [23], in terms of throughput [22], latency [20] and even TCP performance [29] etc. But Wi-Fi Direct based multihop connection setup is getting more attention only in the recent years. D. Camps-Mur et al. first studied the single group Wi-Fi Direct network topology [4], focusing on tethering the $3 \mathrm{G}$ network access to the group clients. Opportunistic group formation is also investigated in the work by M. Conti et al. [9]. The Wi-Fi Direct based content sharing is first brought out by T. Duong et al. in [12]. C. Cassetti et al. designed a connection backbone scheme based on Wi-Fi Direct [7], and focused on the content centric routing performance. It is yet another interesting scheme, which their solution is solely based on two key observations in Android: that broadcast IP packets sent by the GO are always sent through GO's P2P interface whereas unicast packet is invariably sent through the GO Wi-Fi interface. However, neither author claimed these observations hold on any Android platform (later than Android 4.4) nor we can repeat their results in Android 5.0 or later system. C.
Yao et al. also worked on using Wi-Fi Direct to implement the D2D multi-hop network [32]. They assume the network topology will change often, and their data forwarding scheme is based such assumption. However, such scheme may incur high latency and cannot garantee the data will be transmitted to the destination, which is not suitable for our considered scenario.

\section{Applications}

Unlike LBS [24], DiscoverFriends does not need to know the user's location with GPS. The application exploits the broadcast nature of wireless communication to directly find nearby friends without the location information. Also, the user does not expose location information to any server in DiscoverFriends, which completely avoids the potential privacy leak.

In year 2009, a social network application called Safebook [11] was implemented. It adopted a decentralized architecture and capitalized on the trust relationships that existed outside of social networks. Like DiscoverFriends, it addresses the concern where an omniscient service provider such as Facebook or LinkedIn can intercept and potentially monitor interactions between OSN users, essentially nullifying certain privacy policies. Unlike DiscoverFriends however, this application is not based off a MANET environment.

In year 2012, an algorithm for social networking on OLSR MANET utilizing Delayed Tolerant Network (DTN) [28] was implemented. The core idea was to discover friends based on similar interest within the user's neighborhood. The solution realized that the use of Cosine Similarity as the similarity metric yielded the highest number of similar interest matching. Unlike this approach, DiscoverFriends' algorithm has the list of friends already and instead, focuses on how to communicate to them securely assuming they are nearby.

Recently in 2014, an idea to build symmetric private information retrieval (PIR) systems using encrypted Bloom filters was conceived [21]. Rather than putting the user's ID in the Bloom filter as in DiscoverFriends, a RSA signature of each user is placed instead. A client wanting to query a local Bloom filter constructs a blinded query using David Chaum's blinded signature scheme, which then gets signed by the server and passed back. Receiving this, the client proceeds to unblind the query to reveal the server's RSA signature for the targeted client and checks if it exists within its local Bloom filter. However, this approach like DiscoverFriends will fail with a reasonably large user base as the Bloom filter sizes will be too large and inefficient to transmit.

The first peer-to-peer anonymous communication, DC-nets, was proposed in 1988 [8]. However, DC-nets are not scalable and would require each user to transmit several hundred MBs of data to achieve a scheme similar to DiscoverFriends. More recently in 2015 Riposte [10] demonstrated a practical system for anonymous communication. However, Riposte requires several days to complete processing users' reponses as it relies on a seed-homomorphic PRG and expensive elliptic curves.

\section{CONCLUSIONS}

In this paper, we presented DiscoverFriends which is designed to find nearby Online Social Network (OSN) friends without disclosing one's location information to OSN servers, 
eliminating the potential privacy issue in using the Locationbased services (LBS) of OSNs. DiscoverFriends is to our knowledge the first implementaton of a multi-hop Wi-Fi Direct based solution. The use of Bloom filters-based message exchange and a hybrid encryption system with a self-organized public-key management enables confidentiality and authentication while providing higher security by bypassing OSN servers. DiscoverFriends further obscures user ID information by combining multiple OSNs, effectively protecting messages from being identified by any single OSN. By leveraging Function Secret Sharing, users are able to anonymously share their "Check-ins" across multiple OSNs. Under this model, it successfully prevents the effectiveness of eavesdropping in wireless communications as described by the risk analysis under three security threat models.

There are several interesting areas of future work, including

- A more robust mult-hop routing protocol and routing implementation

- A coding scheme to handle collisions for the Function Secret Sharing scheme

- An efficient disruption detection mechanism for handling malformed requests

- A large scale evaluation of Function Secret Sharing

With the design and implementation of DiscoverFriends, we have demonstrated and implemented using Android WiFi Direct how to locate nearby Online Social Network(OSN) friends without violating users' privacy and without disclosing the location information to the OSNs.

\section{REFERENCES}

[1] Number of mobile monthly active facebook users worldwide from 1st quarter 2009 to 3rd quarter 2014 (in millions). http://www.statista.com/statistics/277958/number-of-mobile-activefacebook-users-worldwide/, 2014.

[2] B. H. Bloom. Space/time trade-offs in hash coding with allowable errors. Commun. ACM, 13(7):422-426, 1970.

[3] E. Boyle, N. Gilboa, and Y. Ishai. Function secret sharing. In E. Oswald and M. Fischlin, editors, Advances in Cryptology - EUROCRYPT 2015 - 34th Annual International Conference on the Theory and Applications of Cryptographic Techniques, Sofia, Bulgaria, April 26-30, 2015, Proceedings, Part II, volume 9057 of Lecture Notes in Computer Science, pages 337-367. Springer, 2015.

[4] D. Camps-Mur, A. Garcia-Saavedra, and P. Serrano. Device-to-device communications with wi-fi direct: overview and experimentation. Wireless Communications, IEEE, 20(3):96-104, June 2013.

[5] D. Camps-Mur, X. Prez-Costa, and S. Sallent-Ribes. Designing energy efficient access points with wi-fi direct. Computer Networks 55, no. 13, pp. 2838-2855, 2011.

[6] S. Capkun, L. Buttyn, and J. P. Hubaux. Self-organized public-key management for mobile ad hoc networks. IEEE Transactions on Mobile Computing, pp. 52-64, 2003.

[7] C. Casetti, C. Chiasserini, L. C. Pelle, C. D. Valle, Y. Duan, and P. Giaccone. Content-centric routing in wi-fi direct multi-group networks. CoRR, abs/1412.0880, 2014.

[8] D. Chaum. The dining cryptographers problem: Unconditional sender and recipient untraceability. J. Cryptology, 1(1):65-75, 1988.

[9] M. Conti, F. Delmastro, G. Minutiello, and R. Paris. Experimenting opportunistic networks with wifi direct. In Wireless Days (WD), 2013 IFIP, pages 1-6, Nov 2013.

[10] H. Corrigan-Gibbs, D. Boneh, and D. Mazières. Riposte: An anonymous messaging system handling millions of users. In 2015 IEEE Symposium on Security and Privacy, SP 2015, San Jose, CA, USA, May 17-21, 2015, pages 321-338. IEEE Computer Society, 2015.

[11] L. A. Cutillo, R. Molva, and T. Strufe. Safebook: A privacy-preserving online social network leveraging on real-life trust. IEEE Communications Magazine vol. 47, no. 12, pp. 94-101, 2009.
[12] T. N. Duong, N.-T. Dinh, and Y. Kim. Content sharing using p2psip protocol in wi-fi direct networks. In Communications and Electronics (ICCE), 2012 Fourth International Conference on, pages 114-118, Aug 2012.

[13] Google. Support wi-fi ad hoc networking. https://code.google.com/p/android/issues/detail?id=82, Dec. 2015.

[14] J.-J. Haas, Y.-C. Hu, and K. P. Laberteaux. Efficient certificate revocation list organization and distribution. IEEE Journal on Selected Areas in Communications, vol. 29, no. 3, pp. 595-604, 2011.

[15] M. J. Hinek, S. Jiang, R. Safavi-Naini, and S. F. Shahandashti. Attributebased encryption with key cloning protection. International Journal of Applied Cryptography, vol. 2, no. 3, pp. 250-270, 2012.

[16] I. A. Junglas and R. T. Watson. Location-based services. Communications of the ACM 51, vol. 3, pp. 65-69, 2008.

[17] S. Lee, S. Banerjee, and B. Bhattacharjee. The case for a multihop wireless local area network. In INFOCOM 2004. Twenty-third AnnualJoint Conference of the IEEE Computer and Communications Societies, volume 2, pages 894-905 vol.2, March 2004.

[18] K.-W. Lim, W.-S. Jung, H. Kim, J. Han, and Y.-B. Ko. Enhanced power management for wi-fi direct. In In Wireless Communications and Networking Conference (WCNC), IEEE, pp. 123-128, 2013.

[19] J. Macker and M. Corson. Mobile ad hoc networking and the ietf Mobile Computing and Communications Review, 1998.

[20] D. Maltz, J. Broch, J. Jetcheva, and D. Johnson. The effects of on-demand behavior in routing protocols for multihop wireless ad hoc networks. Selected Areas in Communications, IEEE Journal on, 17(8):1439-1453, Aug 1999.

[21] M. Marlinspike. The difficulty of private contact discovery. https://whispersystems.org/blog/contact-discovery/, Jan. 2014.

[22] P. C. Ng and S. C. Liew. Throughput analysis of ieee802.11 multi-hop ad hoc networks. IEEE/ACM Trans. Netw., 15(2):309-322, Apr. 2007.

[23] H. Nishiyama, M. Ito, and N. Kato. Relay-by-smartphone: realizing multihop device-to-device communications. Communications Magazine, IEEE, 52(4):56-65, April 2014.

[24] K. P. Puttaswamy and B. Y. Zhao. Preserving privacy in location-based mobile social applications. In In Proceedings of the Eleventh Workshop on Mobile Computing Systems \& Applications, ACM, pp. 1-6, 2010.

[25] P. Rathi and P. Mahalle. Certificate revocation in mobile ad hoc networks. International Journal of Application or Innovation in Engineering \& Management (IJAIEM), vol. 2, no. 1, pp. 356-365, Jan. 2013.

[26] K. Sadasivam and T. A. Yang. Evaluation of certificate-based authentication in mobile ad hoc networks. In From Proceeding (464) Networks and Communication Systems, 2005.

[27] K. Sahadevaiah and O. B. V. Ramanaiah. Self-organized public key cryptography in mobile ad hoc networks. Journal of Ubiquitous Computing and Communication.

[28] T. Sanguankotchakorn, S. Shrestha, and N. Sugino. Effective ad hoc social networking on olsr manet using similarity of interest approach. In Internet and Distributed Computing Systems, pp. 15-28, 2012.

[29] K. Tan, F. Jiang, Q. Zhang, and X. Shen. Congestion control in multihop wireless networks. Vehicular Technology, IEEE Transactions on, 56(2):863-873, March 2007.

[30] S. Trifunovic, A. Picu, T. Hossmann, and K. A. Hummel. Slicing the battery pie fair and efficient energy usage in device-to-device communication via role switching. In Proceedings of the 8th ACM MobiCom workshop on Challenged networks, ACM, 2013.

[31] Wikipedia. Firechat. https://en.wikipedia.org/wiki/FireChat, Dec. 2015.

[32] C. Yao, H. Zhang, and L. Song. Demo: Wifi multihop: Implementing device-to-device local area networks by android smartphones. In Proceedings of the 16th ACM International Symposium on Mobile Ad Hoc Networking and Computing, MobiHoc '15, pages 405-406, New York, NY, USA, 2015. ACM.

[33] S. Yi and R. Kravets. Moca: Mobile certificate authority for wireless ad hoc networks. in 2nd Annual PKI Research Workshop (PK103), pp. 65-79, Apr. 2003.

[34] Y. Zhang, W. Liu, W. Lou, and Y. Fang. Securing mobile ad hoc networks with certificateless public keys. IEEE Transactions on Dependable and Secure Computing, vol. 3, no. 4, Oct. 2006.

[35] Y. Zhang, W. Liu, W. Lou, Y. Fang, and Y. Kwon. Ac-pki: Anonymous and certificateless public-key infrastructure for mobile ad hoc networks. In IEEE International Conference on Communications, vol. 5, 2005.

[36] Z. Zhang, W. Susilo, and R. Raad. Mobile ad-hoc network key management with certificateless cryptography. In 2nd International Conference on Signal Processing and Communication Systems, 2008. 